\title{
Home based and hospital based IV antibiotics for cellulitis had similar effects on clinical outcomes
}

Corwin P, Toop L, McGeoch G, et al. Randomised controlled trial of intravenous antibiotic treatment for cellulitis at home compared with hospital. BMJ 2005;330:129.

What is the relative efficacy of home based compared with hospital based intravenous (IV) antibiotics for treatment of cellulitis?

\section{METHODS}

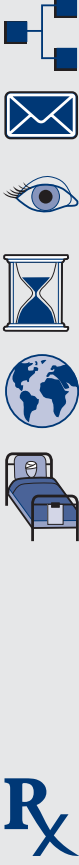

Design: randomised controlled trial.

Allocation: concealed.

Blinding: unblinded.

Follow up period: $\geqslant 4$ weeks.

Setting: metropolitan Christchurch, New Zealand.

Patients: 200 patients $\geqslant 16$ years of age, who presented to the emergency department (ED) with clinical signs of cellulitis and required IV antibiotics because of severity of cellulitis or failure of oral antibiotic treatment; were mentally competent; had a telephone at home and a caregiver nearby; and resided in metropolitan Christchurch. Exclusion criteria included pregnancy, IV treatment for cellulitis of the same site in the previous month, $\geqslant 2$ signs of systemic sepsis (temperature $>38^{\circ} \mathrm{C}$ or $<36^{\circ} \mathrm{C}$, heart rate $>90$ beats $/ \mathrm{min}$, and respiratory rate $>20$ breaths $/ \mathrm{min}$ ) and white blood cell count $>12 \times 10^{9} / \mathrm{I}$ or $<4 \times 10^{9} / \mathrm{I}$ and $>0.1 \times 10^{9} / \mathrm{I}$ immature neutrophils.

D Intervention: all patients received a first IV dose of cephazolin, $2 \mathrm{~g}$, before leaving the ED. 101 patients were allocated to home based treatment (continued IV cephazolin, $2 \mathrm{~g}$, twice daily, administered by community care nurses, and daily visits from a general practitioner). 99 patients were allocated to hospital based treatment ladmission to a hospital ward under the care of the on-call medical team who managed clinical treatment, including choice of ongoing IV antibiotic).

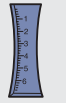

Outcomes: included days to no advancement of cellulitis, days on IV and oral antibiotics, days in hospital or home care programme, and physical functioning and pain (Short Form [SF] 36 questionnaire).

Patient follow up: 194 patients (97\%) (mean age 52 y, 68\% men) were followed up for $\geqslant 4$ weeks.

\section{MAIN RESULTS}

The home based and hospital based care groups did not differ for days to no advancement of cellulitis, days to hospital discharge or on home care, days on IV antibiotics, or days on oral antibiotics (table). The groups did not differ for mean difference in SF-36 scores for physical functioning $(-5.2,95 \%$ CI -13.7 to 3.2$)$ or pain $(-3.8, \mathrm{CI}$ -10.6 to 3.0$)$ at day 6 . For correspondence: Dr P Corwin, Christchurch School of Medicine and Health Sciences, Christchurch, New Zealand. paul.corwin@chmeds.ac.nz Source of funding: no external funding.

\section{CONCLUSION}

Home based and hospital based intravenous antibiotics for treatment of cellulitis did not differ for days to no advancement of cellulitis, days to discharge, days on IV or oral antibiotics, or physical functioning or pain.

Home based $v$ hospital based intravenous (IV) antibiotics for cellulitis*

\begin{tabular}{ll}
\hline Outcomes at $\geqslant 4$ weeks & Hazard ratio $(95 \% \mathrm{Cl}) \dagger$ \\
\hline $\begin{array}{l}\text { Days to no advancement } \\
\text { of cellulitis }(n=180)\end{array}$ & $0.99(0.74$ to 1.34$)$ \\
$\begin{array}{l}\text { Days to discharge }(n=193) \\
\text { Days on IV antibiotics }(n=193)\end{array}$ & $0.95(0.71$ to 1.26$)$ \\
Days on oral antibiotics $(n=194)$ & $0.85(0.64$ to 1.14$)$ \\
\hline
\end{tabular}

${ }^{*} \mathrm{Cl}$ defined in glossary.

thazard ratios $>1$ suggest that home based treatment was faster. Analysis adjusted for age, sex, location of cellulitis (upper $v$ lower limb), and previous antibiotic treatment.

Commentary

M anagement of acute cellulitis by IV antibiotics in primary healthcare settings has often been discussed as an option to alleviate unnecessary hospital admissions. However, until now, little evidence existed to support a change from inpatient management of cellulitis. The study by Corwin et al found similar levels of effectiveness for home based and hospital based treatment of cellulitis.

The study was pragmatic and well conducted. Interestingly, home care patients received only cephazolin, whereas patients treated in hospital were prescribed any antibiotic as determined by clinical preference. Arguably, this suggests that protocol guided drug choice is as effective as that driven by the circumstances of individual cases. A minor quibble is that the extent of presenting cellulitis was marked using an indelible pen - an additional check of baseline comparability would have been to present the average area of cellulitis for each group.

Generally, home IV programmes have been found to compare favourably with inpatient care in terms of cost savings. ${ }^{1}$ The study by Corwin et al seems to be resource intensive, with daily general practitioner visits in addition to twice daily visits by nursing staff, and cost effectiveness cannot be assumed. An economic analysis would have provided useful information for implementation.

Although the groups did not differ for satisfaction with care $196 \% \mathrm{v}$ $96 \%)$, more patients in the home based group than in the hospital based group were satisfied with the location of care $(93 \% \vee 66 \%, p<0.001)$. Such information supports a move towards further development of home based services. The potential for a greater number of patients to be treated in the community by a nurse led service could be explored in further investigations into the development of treatment guidelines in conjunction with medical support.

Anita Latta, RN A+ Links Home Health, Auckland District Health Board Auckland, New Zealand

1 Dalovisio JR, Juneau J, Baumgarten K, et al. Clin Infect Dis 2000;30:639-42. 